Annuaire du Collège de France 2017-2018

\title{
Techniques et économies de la Méditerranée antique
}

Jean-Pierre Brun

\section{(2) OpenEdition}

Édition électronique

URL : https://journals.openedition.org/annuaire-cdf/15888

DOI : $10.4000 /$ annuaire-cdf. 15888

ISBN : 978-2-7226-0572-5

ISSN : 2109-9227

Éditeur

Collège de France

Édition imprimée

Date de publication : 30 décembre 2020

Pagination : 241-268

ISBN : 978-2-7226-0516-9

ISSN : 0069-5580

\section{Référence électronique}

Jean-Pierre Brun, «Techniques et économies de la Méditerranée antique », L'annuaire du Collège de

France [En ligne], 118 | 2020, mis en ligne le 01 avril 2021, consulté le 22 août 2022. URL : http:// journals.openedition.org/annuaire-cdf/15888; DOI : https://doi.org/10.4000/annuaire-cdf.15888 


\title{
TECHNIQUES ET ÉCONOMIES DE LA MÉDITERRANÉE ANTIQUE
}

\author{
Jean-Pierre BRUN
}

Professeur au collège de France

Mots-clés : olivier, huile, techniques, économies, Méditerranée antique

La série de cours et séminaires «L'olivier et l'huile d'olive dans l'Antiquité » est disponible, en audio et en vidéo, sur le site internet du Collège de France (https:// www.college-de-france.fr/site/jean-pierre-brun/course-2017-2018.htm).

\section{ENSEIGNEMENT}

\section{COURS - L'OLIVIER ET L'HUILE D'OLIVE DANS L'ANTIQUITÉ}

\section{Introduction}

Les olives et l'huile d'olive ont été parmi les principaux produits agricoles de l'Antiquité avec les céréales, les légumineuses, le vin et les produits de l'élevage. L'origine de leur emploi et de la domestication de l'olivier remonte au Néolithique au moins. Au fil du temps, ces produits ont fait l'objet de productions domestiques, palatiales ou artisanales, de trafics commerciaux, de prélèvements de tribut et de distributions publiques. Les fluctuations des usages selon les cultures et le développement de l'oléiculture s'inscrivent dans la trame historique tant dans la longue durée que dans les aléas de l'histoire économique et politique. Une première année de cours et de séminaires, en 2017-2018, a été consacrée à un état des connaissances sur l'histoire naturelle, la culture de l'olivier, les techniques de production d'huile et les usages que les Anciens en ont fait. Les origines de la domestication de l'arbre et la diffusion des variétés ont été étudiées à partir des études génétiques et archéobotaniques, permises par les ramassages systématiques de noyaux et de charbons de bois dans les sédiments archéologiques. Ensuite, ont été passées en revue les diverses phases du traitement des olives dont les paramètres botaniques imposent des étapes qui, jusqu'à la technologie moderne, ont peu varié. 
Les usages des huiles, alimentaires ou non, dans les civilisations antiques ont été examinés afin d'en déterminer les paramètres fondamentaux, au regard de nos pratiques contemporaines. Actuellement, nous réservons les huiles pratiquement à la seule alimentation, alors qu'auparavant elles servaient aussi de médicaments, de carburant pour les lampes, de crème pour la peau, de bases pour les parfums, de graisse pour les cuirs, etc. Par la suite seront abordées les évolutions qui affectèrent cette production durant 1500 ans, du début de l'âge du fer à la fin de l'Empire romain. Les essors et les déclins seront étudiés en fonction de la géographie des régions bordant la Méditerranée et des événements politiques et culturels qui les affectèrent.

L'olivier appartient à une famille d'arbres dont six sous-espèces poussent de la Méditerranée jusqu'à la Chine. L'olivier sauvage (Olea europea L. Oleaster) est un arbuste épineux, à petites feuilles, qui produit peu de fruits donnant une huile peu abondante mais délicate. À l'époque gréco-romaine, sa production n'est plus signalée que comme une curiosité, comme base de médicament et de parfum ou pour des usages traditionnels : la couronne du vainqueur des Olympiades était faite des branches de l'olivier sauvage kalistéphanos d'Olympie dont la longévité était extraordinaire selon Théophraste (Recherches sur les plantes, IV, 13, 2).

L'olivier domestiqué (Olea europea L. Sativa) est un arbre qui peut atteindre de grandes hauteurs et porter des fruits de grosseurs diverses suivant les variétés. Celles-ci sont très nombreuses, même sans compter les homonymies; elles ont été créées par l'homme qui a sélectionné les arbres qui donnaient les plus beaux fruits.

C'est au cours de l'ère tertiaire que l'olivier est apparu sur le pourtour de la Méditerranée dans ce continent que l'on appelle «Téthys », où régnaient des conditions climatiques chaudes et humides et donc une végétation tropicale.

Durant le Pliocène, le climat méditerranéen à étés secs et hivers frais se met progressivement en place à la suite des modifications de la circulation atmosphérique et l'apparition du Gulf Stream. Ce climat convient bien aux oliviers qui densifient leurs populations jusqu'à ce que les grandes glaciations de la fin du Pliocène (vers 2,5 millions d'années) et du Pléistocène (entre 1,5 million d'années et 12000 ans avant J.-C.) ne réduisent drastiquement sa distribution dans les zones les mieux protégées du froid sur les bords de la Méditerranée, c'est-à-dire la côte de la Syrie et d'Israël, de l'Afrique du Nord et de la péninsule Ibérique.

Cette répartition dans des zones isolées pendant très longtemps explique la génétique des oliviers sauvages et cultivés. Les oléastres du bassin oriental de la Méditerranée n'ont en effet pas le même patrimoine génétique que ceux de l'ouest. $\mathrm{Au}$ Proche-Orient, les variétés cultivées portent toutes le mitotype ME1, ce qui signifie qu'elles descendent des oléastres locaux. En revanche, le stock génétique des variétés occidentales est hétérogène et s'est formé par hybridation entre les oléastres occidentaux et des cultivars importés d'Orient. L'introduction des cultivars orientaux a été combinée avec la domestication d'oléastres locaux afin de créer des variétés adaptées au climat et aux terrains.

La mémoire génétique des plantes offre donc un support à l'hypothèse d'une domestication de l'arbre à la fois au Proche-Orient et en Occident mais aussi aux transports de boutures dans le bassin occidental de la Méditerranée à partir de l'âge du bronze voire durant le Néolithique.

Après les glaciations, l'olivier s'étend lentement ; l'association entre les pollens d'olivier et de pistachier lentisque devient fréquente à partir du Néolithique, soit dans le courant $\mathrm{du} \mathrm{VI}^{\mathrm{e}}$ millénaire avant $\mathrm{J}$.-C. et l'on commence à trouver des 


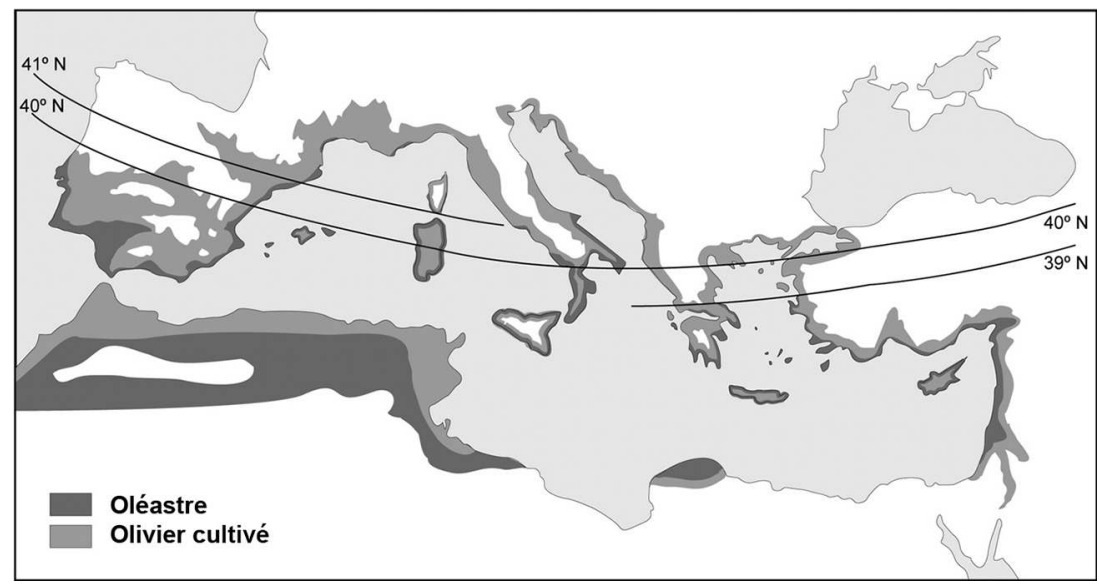

Figure 1 - Répartition de l'olivier sauvage (oléastre) et de l'olivier cultivé dans le Bassin méditerranéen (d'après Carrión, Ntinou et Badal, 2010).

charbons de bois d'olivier dans des gisements de la côte levantine et de la péninsule Ibérique (figure 1).

L'utilisation précoce des arbres sauvages progressivement nettoyés et soignés implique que la culture des arbres fruitiers ait débuté dès la sédentarisation ${ }^{1}$. En même temps que les premières cultures de céréales, les hommes du Néolithique ont commencé à prendre soin d'arbres utiles dont les oliviers qui poussaient sur des terres marginales, dans les garrigues et les collines, où la culture des céréales n'était pas possible, offrant donc une complémentarité et une diversification alimentaires. Comme la récolte des fruits de la vigne et la vinification sont au moins aussi anciennes, on voit que la base de l'alimentation végétale méditerranéenne associant les céréales, le vin, les figues et les olives se met en place dans le courant du Néolithique. Tout au long de l'Antiquité, ce seront les produits de base de l'alimentation : à l'occasion d'un discours prononcé à Antioche en 363 après J.-C., l'empereur Julien opposa au luxe de la table, dans une ville riante et frivole, l'idéal d'une alimentation vertueuse à base de pain, de vin et d'huile (Misopogon 20).

\section{Processus de culture antiques}

Comme pour tous les végétaux, on peut planter des noyaux d'olives pour obtenir des arbres. Zénon de Caunos procéda ainsi pour créer une pépinière dans le grand domaine qu'il gérait dans le Fayoum au $\mathrm{III}^{\mathrm{e}}$ siècle avant J.-C. Ce procédé présente de

1. S.-M. VAlamoti, "Harvesting the "wild"? Exploring the context of fruit and nuts exploitation at Neolithic Dikili Tash, with special reference to wine », Vegetation History and Archaeobotany, vol. 24, n 1, 2015, p. 35-46; C. PAGNOUX, «Fruitiers connus et cultivés en Grèce du Néolithique à l'époque romaine. Confrontation des données archéobotaniques et des sources écrites », Archéosciences, 2019. 
nombreux inconvénients : la pousse est fort lente, le taux de réussite très bas et la variété d'origine n'est pas conservée.

Ces particularités expliquent que le bouturage ait été le mode de reproduction dominant, sous deux formes : branche mise en terre et rejet détaché de la souche considéré par Théophraste (Recherches sur les plantes, I, 8, 6) comme la meilleure technique.

Une autre technique consistait à greffer des rameaux d'olivier sur des troncs d'oléastres. Cette pratique a largement contribué à l'expansion de l'olivier domestique.

Le paysage antique associait donc des oléastres, des oliviers domestiques et des formes hybrides : une bonne illustration est offerte par une inscription de Délos qui, au début du II $^{\mathrm{e}}$ siècle avant J.-C., mentionne, dans le domaine de Thaléon, sur l'île de Myconos, l'association de 200 oléastres, 157 oliviers cultivés et 87 oléastres greffés.

Les oliveraies étaient généralement implantées sur des collines et l'on évitait les fonds de vallée. Les Anciens recommandaient des plantations régulières, mais il y avait des oliviers centenaires qui marquaient le paysage de façon désordonnée. Il s'agissait souvent d'anciens oléastres qui avaient poussé au hasard et qui avaient été greffés (figure 2). Le cas de l'Attique est bien connu : des oliviers sauvages très anciennement greffés et considérés comme sacrés étaient dispersés dans le territoire. Ils pouvaient être mêlés à des oliviers ordinaires dans certaines propriétés comme nous l'apprend Lysias dans le discours Sur l'olivier sacré $(7,24)$, mais ils étaient physiquement séparés des autres par un sêkos, un enclos sacré, qu'on ne pouvait démolir car il était la manifestation de la sacralisation de l'arbre.

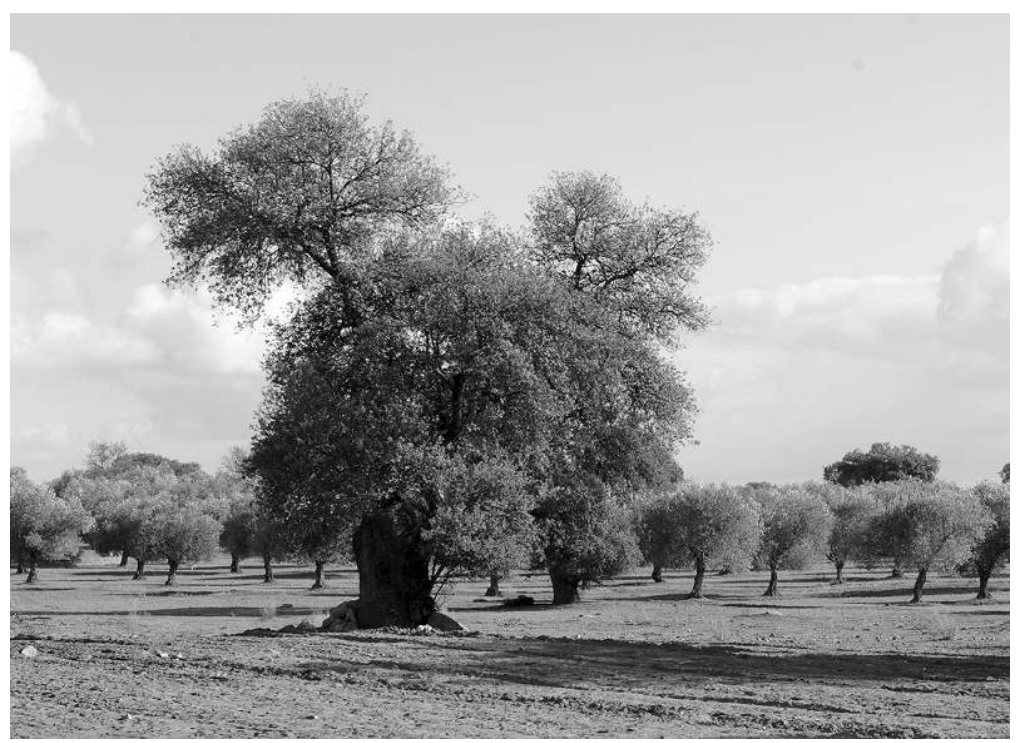

Figure 2 - Olivier pluricentenaire isolé au milieu d'une plantation régulière de jeunes oliviers. Cancho Roano, Espagne (cliché de J.-P. Brun). 
La gestion de ces arbres sacrés appelés moriai était une affaire d'État car ils fournissaient l'huile destinée à récompenser les vainqueurs du concours des Grandes Panathénées qui avaient lieu tous les quatre ans depuis 566 avant J.-C. et qui manifestaient le prestige panhellénique d'Athènes.

A côtés de ces oliviers sacrés, il y avait des arbres ordinaires plantés soit en files, en bordure des chemins et des limites de propriété, soit en rangées régulièrement espacées. Pollux $(\mathrm{V}, 36)$ nomme stoichades des oliviers plantés en file qu'il opposait aux oliviers sacrés dispersés dans la campagne.

Depuis l'époque grecque archaïque au moins, les oliviers étaient utilisés pour marquer les limites de propriété ou de territoire. Par exemple, une inscription de Delphes, datée de 117 avant J.-C., publie une sentence arbitrale délimitant le territoire de cités : ce sont de "vieux oliviers » qui, en un endroit, servent à fixer la frontière entre Delphes et la terre sacrée d'Apollon. Les arpenteurs romains les considéraient aussi comme des marqueurs de limites de propriétés (Hygin, 38). L'archéologie commence à en retrouver des traces : à l'époque romaine, dans l'oasis de Kysis (Tell Douch dans l'oasis de Kharga en Égypte), des champs étaient bordés de files d'oliviers.

Lorsqu'on procédait à la plantation d'oliveraies en rangées, les espacements étaient déterminés en fonction de la pluviosité, de la richesse du sol et de la présence ou non de cultures intercalaires (coltura promiscua). En Grèce classique, les écrits de la classe dominante ne mentionnent pas les cultures intercalaires qui pourraient avoir été surtout pratiquées par les petits paysans. Cela ne signifie pas que les riches propriétaires n'avaient pas de telles cultures: les héritages, les achats, les récupérations de terrains consécutives d'une créance non recouvrée faisaient que les grandes propriétés comportaient des terres de tous types, y compris des cultures intercalaires. Mais les sources dont nous disposons montrent qu'ils préféraient les terrains consacrés à un seul type de culture alors que les petits paysans, par sécurité, complantaient leurs parcelles en céréales et arbres fruitiers dont la vigne et l'olivier. Mais les cultures intensives destinées à la commercialisation impliquaient des arbres plantés régulièrement : dans le discours de Démosthène, Sosithée contre Macartatos $(43,69)$, l'olivette du domaine d'Hagnias semble être plantée de façon dense car elle comprend plus de 1000 oliviers.

Une bonne part de la Méditerranée souffre de déficit de pluviométrie auquel l'olivier résiste tant bien que mal mais qui peut restreindre très fortement sa production, voire le faire mourir : l'arrosage était donc nécessaire dans certaines zones : en Attique, dans les régions arides de Tripolitaine, d'Afrique et de Maurétanie, comme l'indiquent l'inscription de Lamasba et les Tablettes Albertini, voire en Égypte où les lettres de Lucius Bellienus Gemellus donnent des ordres pour irriguer les oliviers de sa propriété située à Dionysias dans le Fayoum vers 100 après J.-C.

La culture proprement dite de l'olivier demande peu d'efforts permanents en dehors de la taille, du piochage et de la récolte, mais les aménagements nécessités par sa plantation sont considérables : les oliviers plantés en plaine demandaient de gros trous de plantation et ceux dans la colline des murs de soutènement de terrasses, des transports de terre des bas de pente, des aménagements de recueil des eaux pluviales. Ces travaux, fort longs et coûteux, ne pouvaient être réalisés que par des paysans ne comptant ni leur temps, ni leur peine pour agrandir leur exploitation sur des terres ingrates et pentues, dégager des profits, certes aléatoires, mais s'ajoutant à ceux plus réguliers des céréales et transmettre cet héritage précieux à leur descendance. 


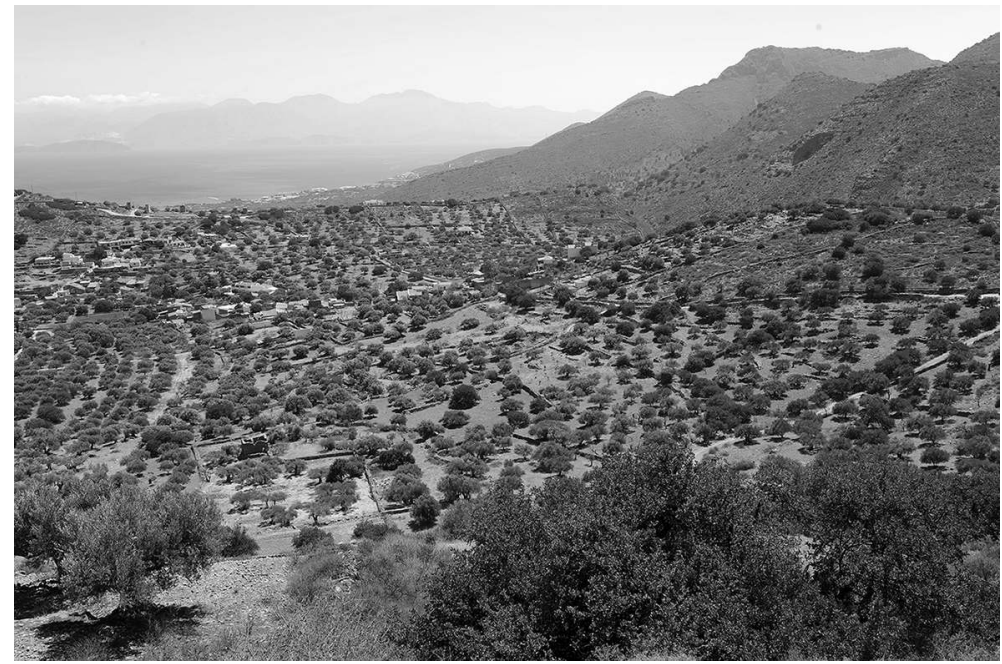

Figure 3 - Oliviers dans des enclos et sur des terrasses dans les collines de Kastelli, Crète (cliché de J.-P. Brun).

En Méditerranée orientale, notamment en Palestine et en Grèce, des murs de soutènement de terrasses et des enclos ont commencé d'être construits dès l'âge du bronze, mais leur continuel remodelage associé à l'imprécision des datations rend très incertain tout discours sur la nature et l'évolution des cultures (figure 3).

La construction des terrasses fut rarement motivée par l'oléiculture et la viticulture, mais bien plutôt corrélée avec la démographie: en Grèce et en Palestine, l'augmentation de la population rendait nécessaire soit l'émigration, soit la création de nouveaux espaces cultivables gagnés sur les pentes. Le plus vraisemblable est qu'on y pratiquait une polyculture vivrière, associée parfois à des cultures arbustives mais sans exclusivité.

Il faut donc renverser les perspectives : les riches propriétaires n'exploitaient pas les terres marginales des collines en construisant des terrasses ; l'investissement en temps et main-d'œuvre n'était pas rentable, d'autant plus que les rendements y étaient bas. Les grandes propriétés comportaient des terres en plaine, faciles à cultiver et plus productives et c'est à elles que s'applique la remarque de Théophraste disant que l'olivier pousse mieux en plaine. La forte proportion des terres détenues par les riches pourrait expliquer la relative rareté des terrasses dans la documentation écrite antique et même dans l'archéologie.

En effet, les terrasses ont toujours été l'affaire de petits paysans qui cherchaient au prix d'un travail harassant et disproportionné à accroître un peu leurs terres cultivables d'autant plus nécessaires pour eux qu'ils avaient des enfants et que la pression démographique augmentait.

Les travaux agricoles que demandait l'olivier en cours d'année étaient relativement limités. Au printemps et en été, on nettoyait les pieds et on arrachait l'herbe comme le montre la célèbre mosaïque des travaux champêtres de Cherchell en Algérie. Durant l'automne, on creusait un bassin autour de l'arbre pour recueillir les eaux de 
pluie. À la fin de l'hiver, on piochait derechef les arbres pour casser la croute afin que l'eau de pluie puisse bien pénétrer ; on en profitait pour apporter de la fumure qui nourrit l'arbre et retient l'humidité. Ce travail de piochage intensif et spécialisé était surtout réalisé dans les propriétés des riches qui disposaient d'esclaves nombreux; les petits paysans qui pratiquaient les cultures intercalaires avaient l'impression que l'olivier ne réclamait pas de travail car il profitait des façons données aux autres cultures.

La taille favorise la pousse du bois de l'année portant les fruits et elle permet d'enlever le bois improductif ou le bois mal placé de façon à ce que l'arbre reçoive de la lumière et de l'air.

\section{La récolte et le traitement des olives}

Le cycle végétatif de l'olivier, qui commence en mars-avril par l'apparition des bourgeons, se poursuit en mai par la formation des grappes de fleurs. De juin à juillet, les fruits se forment et grossissent ; ils commencent à mûrir durant les mois d'octobre et novembre. Les Ménologes rustiques latins du $\mathrm{I}^{\mathrm{er}}$ siècle de notre ère connus par deux inscriptions (CIL I ${ }^{2}$, p. 280) fixent au mois de décembre la cueillette des olives.

Dans l'Antiquité, on tirait de l'olivier plusieurs sortes d'olives à confire et différents types d'huiles. La récolte s'échelonnait donc sur une longue période. Dès les mois d'août et septembre, on ramassait les olives tombées et on cueillait des olives vertes pour les confire ou en faire de l'huile d'été. Selon les régions, en octobre ou en novembre, on cueillait les olives tournantes, c'est-à-dire bigarrées, de couleur verte à violette. Selon Théophraste (Causis plantarum, VI, 19, 3), les olives sont mûres entre octobre et décembre. Il arrivait que, les bonnes années, on cueillît encore en janvier et même en février-mars les olives noires pour les mettre en conserve ou en extraire l'huile « mûre » destinée à l'alimentation.

Tant que les olivettes étaient peu étendues et destinées à couvrir les besoins d'une famille, la récolte pouvait se faire progressivement par ramassage des olives tombées et par la cueillette effectuée le plus souvent par les femmes, les enfants et les esclaves.

Dès que la production augmentait pour déboucher sur un circuit commercial, les agriculteurs devaient s'assurer le concours d'une main-d'œuvre saisonnière. La récolte est en effet un gros travail qui excède les forces de la main-d'œuvre servile présente sur un domaine: les riches propriétaires devaient donc embaucher du personnel supplémentaire ou vendre la récolte sur pied, une pratique qui semble banale au II ${ }^{\mathrm{e}}$ siècle avant notre ère en Italie selon Caton (Agr. 144-146) et en Égypte romaine : un papyrus trouvé à Karanis dans le Fayoum donne le texte d'un contrat d'achat de récolte sur pied durant l'automne 88 après J.-C. (P. Fay. 102).

On pouvait mettre en conserve les olives récoltées, ou en extraire l'huile. Les olives en conserve étaient une nourriture de base pour toutes les classes de la société, et notamment les ouvriers ou les paysans qui les consommaient aux champs avec du pain et des oignons. Dans les Acharniens d'Aristophane (v. 550), les olives sont mentionnées parmi les provisions amassées pour une expédition militaire. Les olives étaient largement consommées par les pauvres et les esclaves : au $\mathrm{II}^{\mathrm{e}}$ siècle avant J.-C., Caton nourrissait ses esclaves d'olives tombées ou d'olives mûres (Agr. 58) et en Afrique, un passage de l'inscription d'Aïn Ouassel (Tunisie) proclame le droit des «colons» d'être dispensés de verser toute redevance sur les olives qu'ils préparaient pour leur consommation personnelle. 
D'un point de vue archéologique, on connaît surtout les conserves d'olives par les étiquettes peintes sur les amphores qui servaient à leur commercialisation outre-mer mais toutes les productions domestiques, de loin les plus courantes, étaient réalisées dans des jarres polyvalentes ou des amphores de remploi.

$\mathrm{Au}$ fil des siècles, le nombre des variétés d'olives reconnues par les auteurs spécialistes d'agronomie a augmenté, reflétant les échanges commerciaux intenses qui se sont développés au cours de la période hellénistique et surtout sous l'Empire romain; ceux-ci furent l'occasion de multiples transplantations de variétés, notamment par greffage, et de la diffusion de la connaissance de ces variétés. Les études archéobotaniques utilisant la morphométrie géométrique confirment l'accroissement du nombre des variétés durant l'Empire romain et permettent de les identifier parmi les variétés connues à l'heure actuelle telles que Koroneiki de Crète ou Lucques.

Les conserves étaient surtout préparées dans les familles mais, sous l'Empire romain, des olives en conserve étaient commercialisées fort loin. On les transportait en général dans des amphores à vin poissées car elles étaient conservées dans du vin cuit (defrutum) ou du vinaigre.

L'une des plus anciennes attestations de ce commerce est l'épave de Cavalière dans le Var. Ce petit caboteur qui a coulé vers 100 avant J.-C. transportant du vin et des olives en conserve qui étaient transportées dans des amphores Dressel 1C et 1A. Plus tard, de nombreuses amphores Haltern 70 originaires de Bétique portent des inscriptions mentionnant des olivae nigrae ex defruto («olives noires dans du vin cuit »). Au $\mathrm{III}^{\mathrm{e}}$ siècle de notre ère, la Crète vendait des olives en saumure dans des amphorettes à fond plat: l'une d'entre elles a été trouvée dans le fort de Dios (désert oriental d'Égypte) : elle porte sur l'épaule l'étiquette Krètikès di'oxou, «olives de Crète dans le vinaigre ». Dans l'épave du Dramont E, un navire venant d'Afrique qui a coulé au large de Saint-Raphaël dans le Var au milieu du $V^{e} s$. de notre ère, de petites amphores cylindriques que les archéologues appellent spatheia contenaient des olives.

\section{L'huile}

Les anciens utilisaient beaucoup de gras animaux, surtout celui de porc, et plusieurs huiles végétales, tirées de graines oléagineuses, que l'on a du mal à saisir dans la documentation archéologique. Toutefois, des analyses chimiques plus fréquentes rétablissent leur juste place à la fois pour l'alimentation mais aussi pour les autres usages tels que l'éclairage ou les soins du corps.

Pour extraire l'huile des olives, point n'est besoin d'un appareillage complexe : un gros galet, une pierre creuse ou un mortier, de l'eau chaude, un linge peuvent suffire.

Pendant longtemps, on a utilisé exclusivement des procédés primitifs qui ne laissent que peu de traces archéologiques et qui ont continué jusqu'à une époque récente à être utilisés pour des productions familiales au Proche-Orient, en Grèce et en Afrique du Nord, ainsi que dans les îles comme la Corse.

Pour les époques archaïque et classique, du VIII ${ }^{\mathrm{e}}$ au IV $\mathrm{IV}^{\mathrm{e}}$ siècle avant J.-C., les moulins et les pressoirs sont peu visibles car ils sont souvent réalisés en bois ou en matières périssables. La construction de grands pressoirs à levier demandait de gros moyens financiers, hors de portée des petits paysans qui formaient la majorité des exploitants. De tels équipements ne se justifiaient que pour de grands propriétaires. Dans les stèles des Hermocopides de 414 avant J.-C., on trouve des mentions de pressoirs à vin et à huile associés à des propriétés ou à des maisons de ville des 
grands propriétaires qui étaient poursuivis pour profanation. Cette répartition socioéconomique très particulière limite la visibilité de l'oléiculture jusqu'à l'époque hellénistique surtout dans les fouilles réalisées anciennement, sans tamisage des sédiments. Seul le tamisage systématique permet de repérer, par la présence de fragments de noyaux d'olives ou de pépins de raisin, des productions dont les instruments n'ont pas laissé de traces indubitables.

\section{Le broyage}

Le broyage des olives était réalisé par plusieurs types d'instruments qu'il faut classer en trois catégories selon le mode opératoire : le broyage par percussion, le broyage par écrasement alternatif et le broyage par écrasement rotatif.

\section{Le broyage par percussion}

a) à bras avec un mortier et un pilon, ce dernier étant le plus souvent en pierre. Ces mortiers sont courants et les archéologues n'ont pas assez prêté attention à eux d'autant plus que leur faible productivité et leur polyvalence s'accommodaient bien de pressoirs à torsion en tissus.

b) au pied, en piétinant les olives avec des sabots de bois. Il était connu en Grèce sous le nom de kroupezai et en latin sous le nom de canalis et solea qu'emploie Columelle (XII, 52, 7), mot à mot «le canal et la chaussure». Il s'agit d'une auge allongée, en bois ou en pierre, dans laquelle l'ouvrier écrasait les olives avec les pieds pour en faire éclater la peau.

c) à l'aide d'un pilon dans une trémie. Columelle (XII, 52, 7) mentionne un appareil qu'il nomme tudicula : il dit qu'elle est semblable à un tribulum qui serait placé verticalement. Le tribulum est une herse à dépiquer le blé qui est formée d'une planche dans laquelle on plantait des éclats de silex. Cette planche était trainée sur l'aire à blé et déchiquetait la paille, libérant le grain. Columelle dit aussi que l'appareil serait relativement efficace s'il n'avait tendance à s'engorger. Cette description implique qu'il s'agit d'un appareil dans lequel les olives sont dilacérées sur une planche hérissée de picots. Il faut donc restituer une trémie comportant une ou plutôt deux planches verticales avec des picots, probablement en silex, dans laquelle on versait les olives ; on devait forcer leur passage avec un pilon et recueillir les olives dilacérées dans un baquet. Ce type d'appareil tout en bois ne laisse généralement pas de trace archéologique.

\section{Broyeurs alternatifs}

La deuxième catégorie regroupe les appareils qui broient les olives avec un mouvement alternatif à l'aide d'un rouleau de pierre que l'on fait rouler dans une auge en pierre à fond plat. Ce mode de broyage est courant en Israël à l'âge du fer (par exemple à Tel Mikné). A-t-il été employé sous l'Empire romain dans les villages du massif calcaire de Syrie du Nord? J'ai avancé dans un ouvrage paru en 2004 que ces rouleaux étaient surtout employés pour écraser les raisins.

\section{Broyeurs rotatifs}

Les premiers broyeurs rotatifs, comportant des meules hémisphériques, furent une véritable révolution technique car ils pouvaient traiter des quantités beaucoup plus importantes de fruits que les procédés primitifs. Les exemples les plus anciens ont été trouvés en Macédoine au IV ${ }^{\mathrm{e}}$ siècle avant J.-C. 


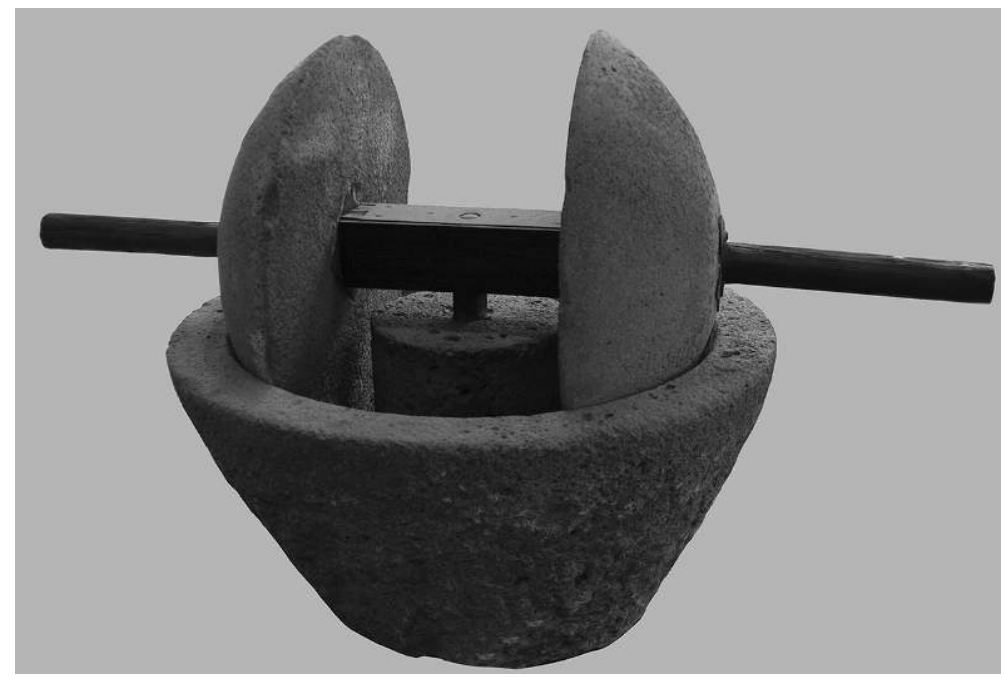

Figure 4 - Trapetum de la villa de la Pisanella, près de Pompéi (cliché de J.-P. Brun).

Trois sous-catégories doivent être distinguées : les moulins à meules verticales hémisphériques, à meules verticales cylindriques et à meules horizontales. Les premiers, que Caton, au début du $\mathrm{II}^{\mathrm{e}}$ siècle avant notre ère, appelle trapeta, se répandirent ensuite à la période hellénistique dans tout le Bassin méditerranéen. On les trouve largement répandus en Grèce, en Italie (c'est par exemple le type exclusif à Pompéi), en Gaule, plus rarement en Espagne et en Afrique du Nord (figure 4). Ces moulins coexistèrent jusqu'à l'époque byzantine avec ceux équipés de meules cylindriques verticales.

La deuxième catégorie comprend les moulins à meules verticales cylindriques : ils sont surtout répandus en Afrique du Nord et en Égypte, mais connus aussi en Italie et en Gaule. Ils peuvent comporter une seule meule ou deux meules de deux types. Le plus courant est une cylindre d'un diamètre important (jusqu'à $1 \mathrm{~m}$ de diamètre) et dont la bande de roulement est étroite (de l'ordre de 10 à $20 \mathrm{~cm}$ ). Un autre type est au contraire formé d'un cylindre de petit diamètre (au maximum $0,50 \mathrm{~m}$ ) mais plus large (de l'ordre de $30 \mathrm{~cm}$ ) ; la bande de roulement peut être lisse ou cannelée afin de pouvoir broyer plus aisément les olives.

On ne connaît pas avec certitude le nom antique du moulin à roues cylindriques verticales. On a longtemps pensé qu'on le nommait mola olearia en interprétant incorrectement le texte de Columelle (XII, 52) : «les meules (molae) font plus de travail que le broyeur trapetum et le trapetum que l'auge et le sabot». Rafael Frankel m'a convaincu que Columelle désigne par mola un moulin à meules horizontales. Il s'ensuit que Columelle et tous les auteurs latins regroupent sous le terme trapetum à la fois le moulin à meules hémisphériques décrit par Caton et le moulin à meules cylindriques : tous les deux broient les olives sous leurs meules roulant verticalement dans un mortier de pierre. 


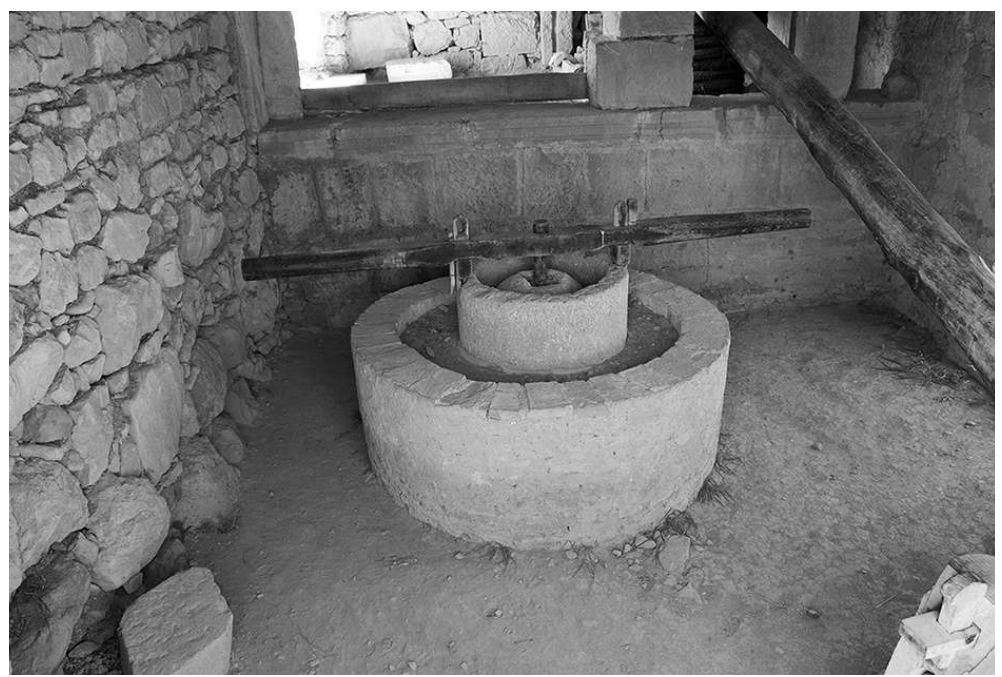

Figure 5 - Mola olearia de Volubilis, Maroc (cliché de J.-P. Brun).

Les moulins à meules horizontales comportent un catillus en forme de couronne, posé sur une meta cylindrique terminée par un cône ou un tronc de cône (figure 5). La meule horizontale est entraînée par un axe poussé par des hommes. Ce type de moulin est omniprésent en Bétique et en Maurétanie tingitane, notamment à Volubilis. Il est totalement absent ailleurs, notamment en Italie.

Ces meules de trapetum ou de mola étaient actionnées par des hommes, le plus souvent des esclaves. L'archéologie n'a pas trouvé de traces de moulins actionnés par des animaux comme on le voit dans les meuneries de grain antiques. J'ai longtemps cherché si l'Antiquité avait utilisé des moulins à huile mus par l'énergie hydraulique. Adapter les machines utilisées pour les moulins à farine pour les transformer en moulins à huile était bien plus aisé que construire des complexes machines à scier utilisant des bielles et des manivelles employées dans les scieries de marbre ${ }^{2}$. Mais parmi les centaines d'huileries antiques dégagées, aucune, même celles appartenant à des domaines alimentés par des aqueducs, n'a livré de moulin à huile actionné par l'énergie hydraulique.

L'explication tient probablement au coût de l'installation et à la courte saison des olivades. Les meuneries à farine ou les bocards à broyer le minerai fonctionnaient toute l'année alors que les moulins à huile ne travaillaient que trois à quatre mois par an. La construction d'une machinerie coûteuse et complexe ne se justifiait probablement pas, sauf dans de très grandes huileries. Mais ces dernières sont le plus souvent situées dans des régions arides comme la Tunisie du Sud ou la Tripolitaine où la disponibilité de l'eau courante posait un problème constant.

2. Voir J.-P. BRUn, «L'archéologie de l'énergie dans l'Antiquité », Annuaire des cours et travaux du Collège de France, vol. 112, 2013, p. 465-490. 
Finalement, il n'y a guère qu'en Bétique que ce progrès technique a pu se répandre : c'est dans cette région qu'on aurait le plus de chance d'en trouver.

\section{Le pressurage}

Après avoir obtenu une pâte d'olive, on la pressait avec divers instruments qui peuvent être classés en cinq catégories.

\section{Les instruments à pression directe}

La pâte d'olive est placée dans des cabas sous des pierres. Ces instruments, les plus anciens, sont les plus difficiles à mettre en évidence archéologiquement. Les installations néolithiques et chalcolithiques taillées dans le roc en Palestine et en Israël ont probablement utilisé de tels procédés. Dans un trou de rocher, on pilait les olives et on mettait sur la pâte des branches avec des pierres, on devait verser de l'eau chaude et récupérer l'huile qui surnageait. Ces procédés primitifs se sont perpétués jusqu'à nos jours dans des contrées reculées du Maghreb comme le Rif marocain où certaines tribus entretiennent quelques oliviers et pressent leurs olives dans des bassines en céramique en les chargeant de plateaux et de pierres.

\section{Les instruments à torsion}

Dès que des tissus, en poils de chèvre notamment, furent disponibles, on put faire des presses à torsion. La pâte d'olives, préalablement broyée, était placée dans un tissu solide que l'on tordait à l'aide de bâtons passés dans des ais. On en trouve des représentations dans les tombeaux égyptiens dès l'Ancien Empire, mais elles concernent surtout le vin et les parfums. Cet instrument simple a connu des perfectionnements : on est passé d'un premier type où les ouvriers tordaient le sac en sens contraire à un second où le sac était placé dans un châssis de bois, rendant l'opération plus efficace. Ce genre de pressoir est invisible archéologiquement mais n'a jamais cessé d'être utilisé pour des petites productions ou des spécialités telles que les huiles à parfum. Durant l'Antiquité, l'emploi de cet instrument a certainement été sous-estimé par les historiens mais il est clair que dès que la production dépassait les besoins domestiques et qu'elle était destinée à la vente, il fallait passer à des appareils plus puissants et encombrants, surtout les pressoirs à levier.

\section{Les pressoirs à levier}

Le principe du pressoir à levier est simple : un tronc d'arbre est fixé d'un côté et abaissé de l'autre. Il écrase contre le sol le marc d'olives généralement placé dans des cabas de sparterie. Leurs traces les plus anciennes remontent à l'âge du bronze sur la côte syro-palestinienne, à Chypre et en Crète : elles sont le plus souvent matérialisées par des maies de pierre, tables circulaires pourvues d'un déversoir.

Les pressoirs à levier se déclinent en six classes : pressoirs à levier simple, actionné par la seule force humaine, pressoirs à levier auquel des blocs de pierre sont suspendus, pressoirs à levier et treuil sur contrepoids de pierre, pressoirs à levier et treuil fixé entre deux montants, pressoirs à levier et vis fixée sur contrepoids et pressoirs à levier et vis fixée sur une poutre de base.

3.1. Les pressoirs à levier simple étaient encore employés dans la seconde moitié du XXe siècle. En Grèce, certains paysans de Thessalie écrasaient la pâte d'olive avec un tronc comportant un plateau circulaire au bout duquel un homme s'asseyait. $\mathrm{Ce}$ genre de pressoir devait être très courant chez les petits paysans à toutes les époques 


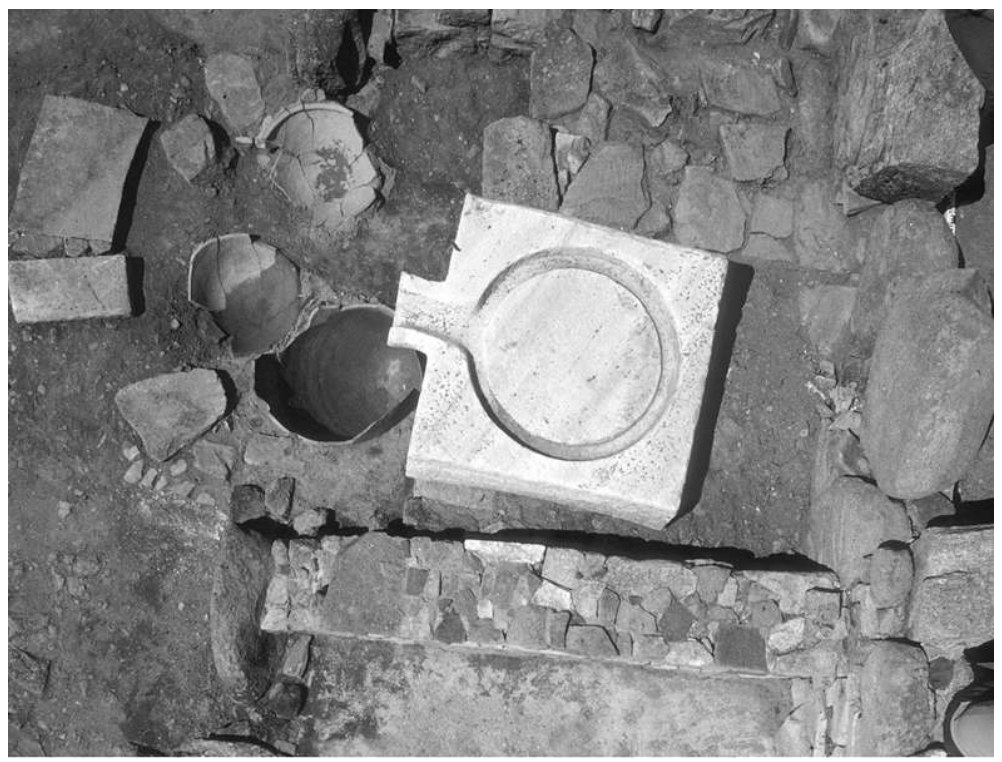

Figure 6 - Pressoir à levier et treuil de Délos, Grèce, avec décantation dans des bassines de terre cuite (cliché de J.-P. Brun).

mais ils sont particulièrement difficiles à mettre en évidence archéologiquement car ils sont formés d'éléments en bois avec parfois une base en pierre.

3.2. Les appareils à levier et blocs suspendus furent très courants en Syrie et Palestine à partir de l'âge du bronze et jusqu'à la fin de l'Antiquité. Un tronc d'arbre était engagé dans une paroi rocheuse ou un mur et on attachait des blocs de pierre en série pour presser la pâte d'olive. À Ugarit, dès le XIII ${ }^{\mathrm{e}}$ siècle avant J.-C., on utilisait déjà des pressoirs de ce type qu'on retrouve en Israël à l'âge du fer par exemple à Tel Mikné, puis à Idalion à Chypre au IV siècle avant J.-C. Les pressoirs souterrains très bien conservés de Maresha en Israël à l'époque hellénistique fonctionnaient encore sur ce principe, de même que certains pressoirs byzantins du Liban.

3.3. Les pressoirs à levier et treuil sur contrepoids sont les plus communs dans les grands domaines du Bassin méditerranéen à partir du $\mathrm{V}^{\mathrm{e}}$ siècle avant J.-C. et surtout sous l'Empire romain (figure 6). Au I ${ }^{\text {er }}$ siècle de notre ère, Héron d'Alexandrie (Mechanika, III, 15) signale l'un des inconvénients de ce type de pressoir : quand le câble se tend à cause du poids du bloc de pierre, le frottement accroît la résistance à un point que les barres de manœuvre du treuil ou le câble lui-même peuvent casser.

3.4. Une autre grande catégorie de pressoir à levier et câble utilise un treuil fixé entre des montants de pierre ou de bois ; elle est très répandue en Italie et en Gaule méridionale sous deux formes :

- les pressoirs dont les deux montants de la tête du pressoir et ceux du treuil sont plaqués au sol par des superstructures maçonnées ; au début du II ${ }^{\mathrm{e}}$ siècle avant J.-C., Caton l'Ancien en a donné une description précise (figure 7); 


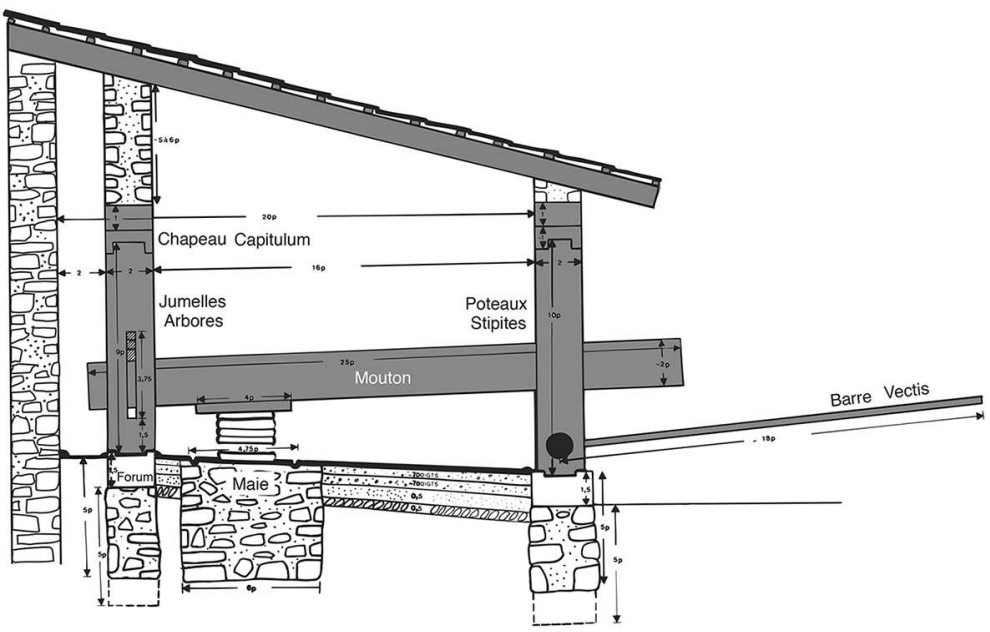

Figure 7 - Pressoir à levier et treuil décrit par Caton l'Ancien (dessin de J.-P. Brun).

- les pressoirs où les montants sont au contraire ancrés dans le sol de la pièce. Ce modèle est celui utilisé à Pompéi et dans les villae de son territoire.

3.5. Les pressoirs à levier et vis furent utilisés en Italie vers la fin du $\mathrm{I}^{\mathrm{er}}$ siècle avant J.-C. au témoignage de Pline qui décrit des appareils dont la vis est lestée soit d'un bloc de pierre, soit d'une grande caisse de bois remplie de pierres. Cette arca lapidum est attestée dans les fouilles récentes de Barcelone où des fosses quadrangulaires contiennent les restes des caisses de bois matérialisées par des frettes de fer et des amoncellements de pierres. La chronologie avancée par Pline l'Ancien (N.H. XVIII, 317) commence à être vérifiée par l'archéologie : quelques pressoirs à levier et vis de Bétique et de Tarraconnaise remontent à la période augustéenne.

Les pressoirs à levier étaient beaucoup plus efficaces que les appareils à torsion ; en revanche, l'adaptation de la vis n'a pas augmenté la puissance des appareils mais, facilitant la manœuvre et en rendant l'appareil plus sûr, elle a permis de gagner du temps de travail et donc d'augmenter la productivité des installations vinicoles et oléicoles. Malgré l'apparition de ces pressoirs à vis plus commodes d'emploi, les pressoirs à levier et câbles se sont maintenus durant toute l'Antiquité. D'une part, les propriétaires d'huileries déjà équipées n'ont pas toujours effectué l'investissement nécessaire pour transformer leur vieux pressoir qui donnait encore satisfaction ${ }^{3}$. Ainsi aucune des huileries des villae ensevelies par le Vésuve en 79 après J.-C. n'utilisait de pressoir à levier et vis. D'autre part, dans des régions où le bois d'œuvre pour tailler les vis était rare, les pressoirs à câbles se sont perpétués : c'est le cas encore aux $\mathrm{IV}^{\mathrm{e}}$ et $\mathrm{V}^{\mathrm{e}}$ siècles de notre ère dans les îles grecques, en Tripolitaine, en Afrique et en Maurétanie.

3. Certains l'ont fait, notamment en Narbonnaise où de nombreux pressoirs à levier et treuil sur contrepoids de pierre ont été transformés en pressoir à levier et vis en adaptant le contrepoids. 


\section{Pressoirs à coins}

Les pressoirs à coins comportent un cadre de bois vertical dans lequel coulissent des traverses horizontales qu'écartent des coins enfoncés à coups de maillet. L'écartement des traverses comprime le marc placé entre la maie et la traverse inférieure. Plusieurs types d'appareils sont connus selon que le cadre de bois est fermé ou fixé dans le sol et selon que les coins sont enfoncés avec des maillets à main ou des maillets suspendus. À l'époque romaine, ces pressoirs étaient surtout utilisés par des parfumeurs: plusieurs fresques de Pompéi et d'Herculanum représentent des parfumeurs au travail dans des ateliers dotés de ce type d'appareil.

Ces pressoirs sont assez difficiles à déceler sur le terrain. J'ai proposé que les deux pressoirs de la parfumerie du Quartier du stade de Délos aient fonctionné avec des coins, mais, à part ce contexte particulier, je n'ai pas identifié d'autres pressoirs de ce type ailleurs. Les pressoirs verticaux de Pompéi, Paestum ou du Fayoum sont, à mon avis, des pressoirs à vis centrale.

\section{Pressoirs à vis centrale}

Dans les presses à vis centrale ou à deux vis latérales, la pâte d'olive n'est pas écrasée par une poutre servant de levier, mais directement par une vis ou par deux vis jumelles, selon le principe de l'étau. L'invention est grecque, peut-être alexandrine. À Alexandrie, au $\mathrm{I}^{\text {er }}$ siècle de notre ère, ces pressoirs étaient assez courants pour que Héron s'en serve comme exemples pédagogiques. Dans le livre III, 19-20 des Mechanika, il décrit deux types d'appareils utilisés pour presser les olives.

Pline l'Ancien, de son côté, donne une description des presses à vis centrale en usage en Italie (N.H. XVIII, 317) et situe précisément leur invention vers le milieu du $\mathrm{I}^{\mathrm{er}}$ siècle après J.-C. Or, vers 30 avant J.-C., Vitruve (De Architectura VI, 6, 3) oppose les pressoirs à levier et treuil aux pressoirs à vis qui tiennent beaucoup moins de place. L'allusion est habituellement rapportée à des pressoirs à levier auxquels on aurait adapté une vis. La lecture du texte ne soutient pas cette interprétation. Vitruve fait cette opposition à propos de l'espace occupé par l'appareil. Or un pressoir à levier et vis est aussi long qu'un pressoir à levier et treuil, d'autant plus qu'ils utilisent tous les deux des contrepoids de pierre. Je pense donc que, contre l'autorité de Pline, il faut admettre qu'il y avait déjà des pressoirs à vis centrale ou à vis latérales de petites dimensions à la fin du $\mathrm{I}^{\mathrm{er}}$ siècle avant J.-C. Rappelons que Vitruve était très au fait des inventions de machines : c'est le premier auteur latin à décrire un moulin hydraulique à roue verticale à augets et il le fait avec assez de précision pour qu'on ne doute pas qu'il en ait vu fonctionner.

La première attestation archéologique bien datée de pressoir à vis centrale est l'appareil carbonisé mis au jour par Amedeo Maiuri à Herculanum. Grâce à des recherches d'archives, Nicolas Monteix a montré que le pressoir publié par A. Maiuri n'était pas une presse de foulon utilisée pour préparer les toges mais bien un pressoir à huile car l'appareil était doté d'une maie en bois et d'une cuve de recueil de liquide.

Il semble que, aussi bien à Pompéi qu'à Herculanum, les pressoirs à vis centrale aient été installés après le tremblement de terre de 62 ou 63 de notre ère, l'évolution technologique profitant de la rupture créée par le séisme pour se diffuser dans les installations nouvellement construites. On en retire donc l'impression que l'archéologie corrobore la chronologie avancée par Pline, mais c'est probablement un biais dû à l'état de préservation des cités ensevelies par le Vésuve. 
Les pressoirs à vis centrale ou latérales ont été reconnus sporadiquement en Occident (Gaule, Italie) surtout dans des installations vinicoles, mais leur structure en bois minore très fortement leur visibilité archéologique. Ils sont plus évidents dans le bassin oriental de la Méditerranée car ils possèdent une base en pierre. On les rencontre souvent à partir du $\mathrm{II}^{\mathrm{e}}$ siècle de notre ère et jusqu'à l'époque byzantine tant pour l'huile que pour le vin.

L'intérêt de ce type de pressoir résidait dans un investissement moindre et dans la possibilité de l'installer dans un espace nettement plus restreint que celui d'un grand pressoir à levier, par exemple dans une boutique. Les inconvénients étaient une puissance inférieure et une moindre rentabilité de la main-d'œuvre dans la mesure où il faut en permanence serrer la vis pour que la pression s'exerce. Ce type d'appareil était donc bien adapté aux exploitations agricoles petites et moyennes et aux ateliers artisanaux urbains, confrontés à des locaux exiguës et disposant de faibles moyens d'investissements mais d'une main-d'œuvre familiale ou servile peu coûteuse.

\section{La décantation}

Broyage et pressurage ne forment qu'une partie de la chaîne opératoire, la dernière opération consiste à séparer l'huile de l'eau de végétation des olives et de l'eau chaude ajoutée pour faciliter l'écoulement de l'huile lors de la seconde pression. Les pressoirs à levier les plus anciens se déversaient dans des vases en terre cuite, puis on leur accola de véritables cuves, soit en pierre de taille, soit, à l'époque romaine, en plomb ou en maçonnerie.

Les vases de terre cuite sont connus à l'époque hellénistique à Délos (III O) et durant tout l'Empire romain en Lusitanie, dans la villa d'Estoi a Milreu par exemple, ou en Bétique dans la fouille récente de Stéphane Mauné à Las Delicias dans la vallée du Genil, un affluent du Baetis par exemple. Ailleurs, comme à Volubilis, on peut avoir de petits dolia qui remplissent cette fonction.

Les cuves de pierre ou de maçonnerie comportaient des aménagements permettant aux opérations de décantation de se réaliser en grande partie sans intervention humaine. Une surverse était ménagée dans la paroi de la cuve recevant le jus de presse, de façon à ce que l'huile, plus légère que l'eau et remontant à la surface, puisse se déverser dans une autre cuve. Ces cuves jumelées sont appelées structile gemellar par Columelle (XII, 52, 10). Caton (Agr. 66) et Columelle mentionnent aussi l'emploi de cuveaux en plomb mis au fond des cuves maçonnées pour y recueillir l'huile par surverse.

Durant la période romaine, les petits bassins étaient généralement complétés par de grandes cuves destinées à la décantation de l'huile des deuxième et troisième pressions durant lesquelles on ajoutait beaucoup d'eau chaude. Il s'agissait de recueillir la plus grande quantité d'huile, servant notamment de combustible pour les lampes. Ces cuves sont fréquentes en Italie (villa de la Pisanella à Boscoreale), en Narbonnaise et surtout en Afrique (Madauros, Thuburbo Maius, Bir Sgaoun, etc.). La suite des opérations de décantation était réalisée à l'aide de récipients en céramique ou en métal.

Les cuves de décantation étaient donc une pièce essentielle du dispositif qui méritait parfois une inscription commémorative. À Rome, sur la via Prenestina, fut retrouvée une cuve monolithe en tuf portant la date de la construction de l'huilerie, 55 avant J.-C., année du célèbre consulat de Pompée et de Crassus. À Tipasa, Marcus 
Hortensius Gaudentius a fait graver son nom et une date correspondant à 278 après J.-C. sur les bassins de décantation.

\section{Le stockage}

Comme pour le vin se posait le problème du stockage de longue durée. Nous savons par les archives que, dès le XXIV e siècle avant J.-C., le royaume d'Ebla entreposait de grandes quantités d'huile à la fois dans les réserves du palais et dans des lieux situés à la périphérie de la ville.

Pour les périodes grecques et romaines, on dispose de textes qui décrivent les conditions de conservation. Selon Théophraste, les celliers doivent être exposés au sud (Causis plantarum, I, 19, 4), une prescription que reprennent les auteurs postérieurs. Au $\mathrm{II}^{\mathrm{e}} \mathrm{s}$. avant J.-C., Caton prévoit d'équiper un domaine modèle de cent dolia olearia, jarres à huile $(A g r .10,4)$. Mais les celliers à huile d'époque romaine retrouvés par l'archéologie sont rares. Dans la villa de la Pisanella à Boscoréale, détruite en 79 après J.-C., cinq dolia à huile étaient déposés dans le couloir d'accès à l'huilerie et quatre autres, rangés contre un mur du chai à vin. L'huilerie de Peymeinade fouillée par Georges Vindry dans les Alpes-Maritimes possédait des salles contenant 14 jarres d'une contenance totale de 5000 à 7000 litres. En Syrie du Nord, à la fin de l'Antiquité, il arrivait que l'on conservât l'huile dans des cuves taillées dans le rocher, au fond d'un entrepôt souterrain comme à Kafr Nabo.

Les villes, pour la régularité de leur ravitaillement, se devaient de posséder de grands entrepôts. Citons par exemple les Horrea Galbae : en 175 après J.-C., une inscription rappelle que C. Pomponius Turpilianus était procurator ad oleum de ce lieu et du port d'Ostie. En 373 après J.-C., à Carthage, l'entrepôt de l'annone s'appelait conditiorum Zeugitanum et on y conservait l'huile dans des amphores, notamment dans de grands conteneurs cylindriques de plus de 145 litres et dans de plus petits, nommés centenaria, d'une contenance moyenne de 36 litres.

\section{Le transport}

Comme pour le vin, le plus ancien récipient de transport de l'huile fut l'outre. En général tirée d'une peau de chèvre ou de bouc, elle pouvait être faite aussi de plusieurs peaux de bœuf cousues ensemble lorsqu'on voulait réaliser un grand conteneur.

Les outres sont attestées en Mésopotamie, en Égypte, en Grèce et, encore sous l'Empire romain, elles sont le récipient le plus couramment employé pour le transport de l'huile par voie de terre ou à courte distance. En Apulie, au I ${ }^{\text {er }}$ siècle avant J.-C., ce sont des troupeaux d'ânes qui transportaient l'huile et le vin de l'intérieur jusqu'au port de Brindes, évidemment dans des outres (Varron, $R$. $R$. II, 6, 4). En Égypte, en 114 après J.-C., un papyrus ( $P$. Wisc. II, 80) indique que l'huile contrôlée à la douane était transportée en outres d'environ 50 litres ${ }^{4}$. Le tarif de l'octroi de Palmyre, daté du 18 avril 137 après J.-C., indique qu'en Syrie romaine, l'huile d'olive était transportée dans des outres à dos de chameaux ou d'ânes.

La place des récipients en peau est sous-estimée par la recherche archéologique car, à la différence des amphores, ils ne se conservent pas - faisons abstraction des

4. Ph. MAYERSON, « Measures (metrètai) and donkeyloads of oil in P. Wisc. II, $80 »$, Zeitschrift für Papyrologie und Epigraphik, vol. 131, 2000, p. 189-192. 
cas exceptionnels : certains gisements du nord de l'Europe noyés dans la nappe phréatique, ou, à l'inverse, les sites des pays désertiques (Syrie, Égypte). Or les ostraca trouvés dans l'îlot de l'Amirauté à Carthage, une trentaine de reçus datant de l'année 373 après J.-C., montrent que plus de la moitié de l'huile collectée par l'État romain au titre de l'impôt en nature était livrée dans des outres d'une contenance de 72 livres (26 litres) et surtout dans de grands récipients en peau contenant 504 livres d'huile, soit 182,7 litres. Ces grands récipients étaient placés sur des chariots que l'on utilisait comme wagons-citernes. L'huile était ainsi convoyée jusqu'au port où, après une opération de pesage, elle était transvasée dans des amphores qui n'étaient systématiquement utilisées que pour les transports par bateaux. Au cours des trois premiers siècles de notre ère, on procédait de cette façon pour expédier vers Rome et les armées du Rhin l'huile produite en Bétique : transportée dans des outres depuis les huileries jusqu'aux ports, l'huile était alors versée dans des amphores. Quelquefois, néanmoins, on employait aussi les amphores pour les trafics terrestres, soit qu'on les charge deux par deux sur des ânes, soit qu'on les place sur des chariots. Le cas de Carthage est caractéristique : l'huile destinée au fisc arrive de la région d'Hippone en amphores transportées par bateaux, tandis que celle provenant de l'intérieur de la Zeugitane est livrée dans des outres.

Dans les villes méditerranéennes, la plus grande partie de l'huile produite localement était donc apportée dans des conteneurs en peau. Les amphores que les archéologues retrouvent témoignent donc essentiellement des importations. Autant dire que les statistiques auxquelles elles donnent lieu sont faussées à la base, puisque la majeure part de la consommation, celle satisfaite par les producteurs locaux ou régionaux, échappe à toute quantification.

Certaines zones de la Méditerranée furent exportatrices d'huile sur la longue durée : les côtes de l'Adriatique, l'Espagne, l'Afrique, les rives de la mer Égée. Ces régions ont produit des amphores destinées au commerce de l'huile depuis l'époque archaïque jusqu'à l'Antiquité tardive afin de répondre à la demande d'huile de régions qui en manquaient.

Ces régions d'importation présentent des caractéristiques communes. Il n'y a d'exportation d'huile que vers les lieux où existe une demande culturelle forte car l'huile d'olive n'est pas indispensable : le gras animal ou d'autres huiles végétales sont plus couramment employés dans des communautés telles que les Celtes ou les Égyptiens.

L'huile d'olive n'était importée massivement que dans deux cas : lorsque des groupes humains la réclamaient dans des zones situées hors de l'aire méditerranéenne et lorsque la demande urbaine excédait les capacités de production du territoire économique d'une ville.

Dans ce premier cas, l'huile d'olive est un marqueur culturel que demandent par exemple les colonies grecques implantées dans des zones non productrices telles que le nord de la mer Noire. Au II $^{\mathrm{e}}$ siècle avant notre ère, Polybe (IV, 38, 5) indique qu'elles importaient de l'huile en échange de miel, de salaisons de poissons, de bestiaux et d'esclaves. Il écrit que les Grecs ne pouvaient pas être privés de toutes ces denrées et que les habitants de Byzance tiraient un grand profit de ce commerce. Un autre type de consommateur culturellement déterminé est le soldat italien ou méditerranéen. Des marchands approvisionnaient en huile d'Espagne, d'Afrique, d'Istrie ou de mer Égée les troupes romaines stationnées dans le nord de l'Europe, sur le Danube ou dans des lieux isolés comme les déserts. C'est ainsi qu'aux ${ }^{\mathrm{er}}$ et $\mathrm{II}^{\mathrm{e}}$ siècles de notre ère, les militaires en poste au Mons Claudianus ou à Didymoi 
dans le désert Oriental d'Égypte commandaient, tout comme leurs homologues de Vindolanda en Bretagne ou de Haltern ou Hofheim en Germanie, de l'huile d'Espagne (dans des amphores Dressel 20), d'Afrique (dans des amphores Africaines 1), de Tripolitaine (dans des amphores Tripolitaine 1) et de la mer Égée (dans des amphores Dressel 24).

Dans le deuxième cas, la demande d'huile était provoquée par l'augmentation démographique de villes devenues capitales régionales ou impériales. Leur croissance était telle qu'il fallait importer massivement toutes sortes de produits alimentaires (céréales, salaisons de poissons et de viande, vin, huile). Dans le cas de Rome, puis de Constantinople, il fallut organiser toute une filière d'approvisionnement avec des incitations fiscales pour les marchands afin d'éviter les pénuries car la consommation dépassait de loin les productions régionales d'huile qui restent peu visibles pour nous car elles étaient apportées en outres.

D'autres exemples sont significatifs. Marseille grecque, dotée d'un petit territoire, a dû importer de l'huile d'Espagne dès le $\mathrm{VI}^{\mathrm{e}}$ siècle avant J.-C. pour subvenir à ses besoins. Sur la longue durée, la ville d'Alexandrie aussi est emblématique : sa population nombreuse, en partie composée de descendants de colons grecs, demandait de l'huile d'olive et dédaignait l'huile de graine locale. Or Strabon (17, 1, 35) écrit que, mis à part le Fayoum, «L'Égypte ne possède pas d'olivier à l'exception des vergers proches d'Alexandrie, qui peuvent donner des olives mais ne fournissent pas d'huile. » Il témoigne donc d'oliviers produisant des olives pour la conserve mais pas pour l'huile. La thèse de Valérie Pichot intitulée Aux portes d'Alexandrie. Le développement de la Maréotide hellénistique et romaine (2017) a montré qu'il y avait très peu d'huileries dans la région au sud-ouest d'Alexandrie. Celles qui ont été retrouvées en fouille sont datables de l'Antiquité tardive. Alexandrie importa donc au cours de son histoire de l'huile de Brindes, puis d'Espagne, d'Afrique et de Tripolitaine, outre celle du Fayoum, sans pouvoir d'ailleurs faire face complètement à la demande puisque, selon le Talmud (Shabbath 26a), la seule huile aisément disponible à Alexandrie était l'huile de radis, oleum raphaninum, dont Pline et de nombreux papyrus indiquent effectivement que l'Égypte en produisait beaucoup.

Ces particularités de l'offre et de la demande expliquent que les types d'amphores à huile soient nettement moins nombreux que ceux d'amphores à vin ou à salaisons de poisson. Ces deux produits étaient commercialisés en bien plus grande quantité et diversité que l'huile pour deux raisons.

D'une part, l'huile est un produit concentré qui est extrait en petite quantité et qui est consommé pour la nourriture et les soins du corps en proportions plus réduites que le vin ou les conserves de poisson. La demande est peu élastique à la différence du vin qui est avant tout une drogue dont la consommation est très extensible. Il est donc normal que les amphores à huile soient nettement moins nombreuses que les autres, sauf exception comme l'approvisionnement organisé de la ville de Rome qui a produit une montagne de tessons d'amphores à huile d'Espagne, le Monte Testaccio.

D'autre part, les variétés de vin et de produits à base de poisson sont bien plus nombreuses que celles de l'huile dont le commerce porte surtout sur l'huile d'olive et dans une bien moindre mesure sur certaines huiles de graines comme le ricin pour les lampes.

Par conséquent, on constate à la fois la grande variété des amphores à vin et à poisson mais aussi des différences de taille : certains vins de prix étaient en effet exportés dans de petits conteneurs, cruches, amphorettes, ce qui ne semble pas avoir été le cas de l'huile ; les vins communs étaient expédiés dans de grandes amphores, 
puis surtout des tonneaux, de même que les salaisons de poisson. L'huile d'olive non parfumée n'était pas un produit de luxe, ce qui explique qu'il n'y ait pas d'attestation de trafic en petits conteneurs, même si les anciens savaient apprécier ses différentes qualités qui varient selon le degré de maturité, le mode de détritage, la phase de décantation ou la région d'origine.

\section{Les usages de l'huile}

L'huile d'olive jouait certes un rôle alimentaire, mais elle servait aussi et beaucoup aux soins du corps, à la parfumerie, à la pharmacie, aux rituels, à l'artisanat textile, à l'éclairage, au graissage. Les plus anciens usages attestés ne concernent d'ailleurs pas l'alimentation mais la parfumerie et, d'une façon plus large, les soins du corps et la médecine.

\section{Soins du corps}

Depuis les temps les plus reculés, les corps gras animaux et végétaux ont été utilisés pour protéger et assouplir la peau. Les anciens Mésopotamiens et Égyptiens se servaient essentiellement d'huile de sésame. Mais les Méditerranéens employaient surtout l'huile d'olive : ce sont des jarres d'huile d'olive et des flacons d'huile parfumée au myrte et à l'oliban que les rois d'Ebla en Syrie envoyaient en cadeau à ceux de Mari dans la seconde moitié du III' millénaire avant J.-C. L'onction d'huile, parfumée ou non, était le complément normal du bain depuis l'âge du bronze : on en trouve continûment des témoignages depuis les épopées homériques jusqu'à la fin de l'Antiquité avec l'extraordinaire développement des établissements thermaux où l'on pratiquait des massages.

Pendant l'époque hellénistique et romaine, dans tout l'Orient, on fit une grande consommation d'huile au gymnase et à la palestre. Les athlètes s'en enduisaient le corps avant la lutte ou la course. Elle les protégeait des ardeurs du soleil et fixait la poussière dont ils se recouvraient. L'exercice fini, ils raclaient l'huile et la poussière avec de longues cuillères en bronze, les strigiles, puis nettoyaient leur peau avec de l'eau, de la potasse, de la soude ou du nitre à l'aide d'une éponge. Ils passaient ensuite dans l'étuve afin de finir de se décrasser en suant, puis calmaient et assouplissaient leur peau irritée en se faisant masser avec de l'huile chaude.

À l'époque classique et au début de l'époque hellénistique, on chercha les moyens de garantir l'huile aux gymnastes ${ }^{5}$. L'huile était fournie par les cités ou par les usagers du gymnase qui se cotisaient pour financer la dépense. Il arrivait que le gymnase soit doté de terrains ou de locaux qui étaient loués et de fonds qui étaient prêtés. Un Lycien nommé Tlépolémos offrit des terrains à Delphes pour la fourniture de l'huile à la fin du $\mathrm{III}^{\mathrm{e}}$ siècle et, vers 160 , il y eut une souscription civique à Élatée (SEG 42 [1992], 478) dans ce but.

À partir de la seconde moitié du $\mathrm{II}^{\mathrm{e}}$ siècle avant J.-C. puis durant les premiers siècles de l'Empire, ce furent les gymnasiarques qui furent chargés de la fourniture de l'huile. Au début, les gymnasiarques assuraient l'approvisionnement en huile mais ne l'offraient pas de leurs deniers mais, vers la fin du $\mathrm{II}^{\mathrm{e}}$ siècle, des inscriptions commémorent la

5. P. FRÖHLICH, «Les activités évergétiques à l'époque hellénistique tardive : la fourniture de l'huile », in O. CURTY (dir.), L'Huile et l'Argent. Gymnasiarchie et évergétisme dans la Grèce hellénistique, Paris, De Boccard, 2009, p. 57-94 
générosité de certains gymnasiarques tel un certain Philippos qui, en 106/105 avant J.-C., refusa d'utiliser l'argent réuni par les usagers et paya lui-même l'huile.

Outre l'huile pour les gymnastes, le gymnasiarque offrait parfois de l'huile pour le bain. La première attestation concerne Zôsimos de Priène qui paya l'huile pour oindre les baigneurs après le bain et élargit cette libéralité aux étrangers venus pour la panégyrie. Désormais les gymnases sont dotés de thermes et seront bientôt absorbés par eux.

La consommation de l'huile dans les établissements de sport était donc considérable : entre 195 et 167 avant J.-C., la cité de Tauromenion en Sicile attribua 3800 litres d'huile par an à son gymnase. À la fin de l'époque hellénistique et sous l'Empire, les gymnasiarques s'engageaient donc dans d'énormes dépenses que seuls les plus riches pouvaient se permettre.

Ces fournitures gratuites d'huile ne pouvaient guère se concevoir que dans des régions où l'on en produisait en abondance. Remarquons que les inscriptions sont concentrées sur les bords de la mer Égée, en Grèce de l'Est, en Sicile, mais aussi dans les Alpes Maritimae et en Narbonnaise où quelques inscriptions mentionnent des distributions d'huile faites par des notables locaux dans le courant du II $^{\mathrm{e}}$ siècle après J.-C.

L'usage intensif de l'huile pour les massages après le bain a provoqué en Italie et dans toutes les provinces une hausse de la consommation d'huile. La multiplication des thermes privés et surtout publics qui, à partir du $\mathrm{II}^{\mathrm{e}}$ siècle, deviennent les monuments publics les plus nombreux et les plus fréquentés s'est accompagnée nécessairement d'une augmentation considérable de l'huile, parfumée ou non, qu'on y utilisait.

\section{Médicament}

Parallèlement à son rôle dans les soins du corps, l'huile servait de médicament. L'huile était utilisée soit seule, soit comme excipient. Dioscoride (I, 30 ; I, 105) et Pline (N. H. XXIII, 77) recommandent l'huile d'oléastre et l'omphacium en bain de bouche contre les gencives putrides.

Les raclures d'huile, de sueur et de poussière que les athlètes enlevaient de leur corps après s'être exercés à la palestre étaient réputées avoir des vertus médicinales. En grec, on l'appelait gloios. Selon la loi de Beroia, vers 180 avant J.-C., le gardien du gymnase, un esclave, entretient les lieux ; il reçoit en échange le gloios. Ailleurs, ces raclures d'huile étaient revendues avec profit par les directeurs de gymnases (Pline, N. H., XV, 19). Le gloios était en effet vendu aux pauvres pour s'en enduire ou bien on en faisait des remèdes utilisés en gynécologie et en rhumatologie (Dioscoride I, 34 ; Pline, N. H., XXVIII, 50). L'huile enfleurée à la rose ou à l'œnanthe était censée combattre les poisons, les ulcères, apaiser les coliques, chasser les vers intestinaux, faire tomber la fièvre, etc. Pratiquement toutes les huiles parfumées avaient des indications thérapeutiques ${ }^{6}$.

\section{Alimentation}

Dans les pays de montagne, c'était le gras animal, surtout le lard et le saindoux, qui servait de fond de cuisine. Pour la Grèce classique, le corpus hippocratique

6. Voir J.-P. BRUN, « La production des parfums dans l'Antiquité. L'apport des analyses, de la céramologie et de l'épigraphie à l'étude des parfums antiques », Annuaire des cours et travaux du Collège de France, vol. 113, 2014, p. 465-485. 
indique que les légumes sont assaisonnés à l'huile, au fromage, au miel, à l'oignon, au vinaigre, au silphion, une plante aromatique qui poussait sur les côtes de Lybie. Hippocrate considérait que l'huile est un produit de luxe, utile en petite quantité, nuisible en excès de consommation alors que l'usage externe était toujours valorisé pour l'exercice et pour les massages après le bain. Cette observation suggère que les riches consommaient beaucoup d'huile et que les médecins tentaient de les réfréner (Hippocrate II, 58).

Les sources juives apportent des informations d'un autre ordre. La Michnâh (traité Ketubot 5, 8-9) indique la ration minimale de vivres que doit recevoir une femme lorsque son mari s'absente pour une semaine; cette ration comprend: deux kilogrammes de blé, des pois chiches, des lentilles, des olives en conserve et de l'huile d'olive.

Les Romains utilisaient l'huile d'olive comme fond de cuisine. Un passage de la pièce de théâtre Pseudolus de Plaute, jouée pour la première fois en 191 avant J.-C., fait allusion à un ragoût à base de viande qui pourrait être rendu unctiusculus - c'està-dire onctueux, bien gras - par ajout d'huile d'olive.

Le livre de cuisine attribué à Marcus Gavius Apicius, un riche personnage de l'époque d'Auguste et Tibère, mais en réalité compilé au IVe siècle à partir de recettes plus anciennes, mentionne l'huile d'olive dans la plupart des préparations, mais cette omniprésence de l'huile d'olive n'atteignait probablement pas les campagnes car le traité incarne la cuisine raffinée, réservée aux élites urbaines.

\section{Éclairage}

Les premières lampes à huile connues remontent au Chalcolithique. Leur usage se répandit ensuite très rapidement dans toutes les agglomérations du Proche-Orient, où, selon les lieux, les lampes brûlaient de l'huile de graine ou d'olive. À Ougarit, aux $\mathrm{XIII}^{\mathrm{e}}$ et $\mathrm{XII}^{\mathrm{e}}$ siècles avant J.-C., la production d'huile d'olive tenait une place considérable et les archives attestent qu'elle était employée autant pour l'alimentation que pour l'éclairage, notamment des temples.

En Grèce, la lampe à huile détrôna la torche pour l'éclairage nocturne des temples et des maisons dès l'époque classique. Au IV siècle, lorsqu'on organisait un banquet entre amis, l'hôte fournissait l'huile des lampes pour l'éclairage (Théophraste, Caractères, XXX, 18). La mèche qui trempait dans l'huile et sortait par un bec pouvait brûler environ deux heures et consommait 0,5 centilitre par heure. Mais cette faible consommation d'huile de seconde qualité est à relativiser car, pour éclairer correctement une pièce, il fallait de nombreuses lampes ou des lampes à plusieurs becs qui se multiplient à partir de l'époque hellénistique (figure 8 ).

À Rome, les lampes étaient remplies avec des huiles de qualité inférieure qui étaient mélangées avec du sel. Le sel absorbait l'humidité et donnait à la flamme une couleur jaune. On brûlait toutes sortes d'huiles et de gras dans les lampes, notamment de l'huile de ricin (Pline, $N$. H., XV, 25). Les analyses biochimiques ont montré que cinq amphores africaines trouvées dans un entrepôt de Classe (Ravenne, Italie) contenaient de l'huile de ricin destinée à l'éclairage.

Mais cet usage de l'huile dans les lampes valait surtout pour les régions de production: dans les zones dépourvues d'oliviers ou d'huiles de graines, on l'épargnait et on ne s'éclairait guère. 


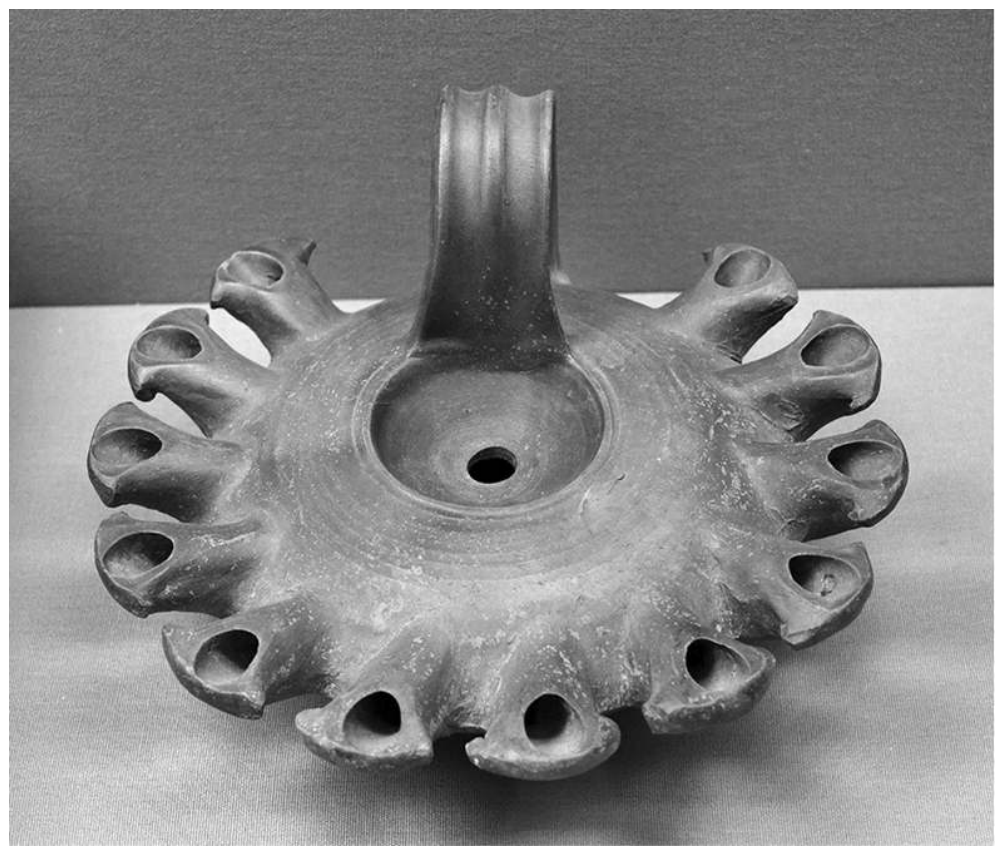

Figure 8 - Lampe à huile à douze becs fabriquée à Cnide au II ${ }^{\mathrm{e}}$ siècle av. J.-C. British Museum (cliché de J.-P. Brun).

\section{Usages religieux}

L'huile était offerte aux dieux sous diverses formes, notamment en libations sur l'autel. Il fallait remercier les dieux de leur don en leur offrant une part des récoltes. Ces pratiques sont attestées dès le XXIV e siècle avant J.-C. dans les archives d'Ebla.

Les statues de cultes étaient enduites d'huile. En Grèce, on oignait d'huile, parfumée ou non, les statues de culte, comme l'omphalos de Delphes (Pausanias, Périégèse, X, 24, 6) ou les statues du sanctuaire de Délos. Les particuliers procédaient de la même façon avec leurs génies domestiques et avec les autels placés dans les rues. Le seul fait d'enduire d'huile une pierre en faisait une pierre sacrée d'où les rituels employés pour les bornes délimitant les champs qui deviennent ainsi inviolables (Siculus Flaccus, De Conditione Agrorum, 106).

\section{Rites funéraires}

Un aspect particulier concerne l'utilisation de l'huile lors des funérailles et des rites funèbres. Lors du sacrifice funéraire en l'honneur des Platéens morts durant les guerres médiques, on faisait des libations de vin, de lait et on apportait des fioles d'huile et de parfums pour oindre les stèles funéraires (Plutarque, Aristide, 52). Avant la crémation ou l'ensevelissement, on oignait le corps d'huile parfumée et d'onguents dans lesquels entrait l'huile d'olive (Virgile, Énéide, VI, 219 ; Apulée, Florides, IV, 19). 


\section{Les sous-produits de la fabrication de l'huile d'olive : margines et grignons}

Les margines sont les résidus liquides de l'extraction de l'huile, les latins l'appelaient amurca, et les grignons sont les résidus solides : les noyaux et la pulpe pressée, les latins l'appelaient simplement nuclei olivarum.

\section{L'amurca}

Les Anciens faisaient un emploi très vaste des margines, résidus du pressurage mêlant l'eau de végétation des olives et des matières grasses en suspension. En la chauffant, on éliminait la partie aqueuse. Ces traitements permettaient d'obtenir un liquide huileux utilisé comme insecticide, en traitement phytosanitaire et comme graisse pour les cuirs et les métaux. En médecine vétérinaire, l'amurca était utilisée comme fortifiant pour les bœufs, contre la gale des moutons ou pour éliminer les puces des chiens.

\section{Les grignons}

Après le passage à la presse et extraction de l'huile, le résidu solide est appelé « grignons ». Il s'agit d'un mélange de noyaux d'olives écrasés et de pulpe asséchée. Une tonne d'olives donne 200 litres d'huile, 450 litres de margines et $350 \mathrm{~kg}$ de grignons, un combustible remarquable à la fois par son pouvoir énergétique et par ses qualités de combustion. Les grignons ne dégagent pratiquement pas de fumée et ils étaient donc très prisés pour chauffer les maisons antiques dépourvues de véritables cheminées et employant des braseros. On les utilisait couramment pour chauffer les fours de potiers (à Athènes, à Leptiminus, par exemple) et de métallurgistes, voire les fours de boulangers : à Pompéi, ces derniers étaient chauffés à plus de $90 \%$ par des grignons, ce qui s'explique par la saison à laquelle l'éruption se produisit : le temps des olivades et de l'extraction de l'huile d'olive ${ }^{7}$.

\section{SÉMINAIRE}

\section{Séminaire 1 - L'archéobotanique de l'olivier}

Jean-Frédéric Terral (professeur à l'université de Montpellier), le 7 novembre 2017

Ce séminaire a fait le point des recherches actuelles sur la botanique de l'olivier et son développement sur le long terme en tant qu'arbre exploité puis domestiqué et cultivé. Les méthodes d'investigations utilisent la carpologie et l'anthropologie avec des raffinements récents tels que l'éco-anatomie quantitative et la morphométrie géométrique. Cette méthode consiste à prendre un grand nombre de mesures sur les noyaux d'olives trouvés dans les sédiments archéologiques afin de caractériser les variétés par comparaison avec les exemplaires actuels. Elle permet d'identifier les cultivars utilisés à diverses périodes et donc à les rattacher à des familles désormais bien caractérisées par leurs rendements et leurs qualités organoleptiques. Travaillant aussi sur les bois, l'équipe de l'université de Montpellier a pu prouver l'existence de

7. N. MonteIX, «Pompéi, recherches sur les boulangeries de l'Italie romaine », The Journal of Fasti Online, 2009 (http://www.fastionline.org/docs/FOLDER-it-2009-168.pdf) 
la taille de l'olivier à l'époque néolithique et donc une forme de culture et même de l'irrigation, notamment au Moyen Âge. Au total, l'histoire naturelle de l'olivier restituée à partir des échantillons prélevés dans les sédiments archéologiques concourt à écrire une histoire de la diffusion de l'oléiculture plus ancienne et plus complexe que les théories traditionnelles : ce ne sont pas les Phéniciens et les Grecs qui ont apporté cette culture en Occident, du moins pas partout. L'olivier était déjà exploité depuis le Néolithique dans la péninsule Ibérique, en Afrique et depuis au moins l'âge du bronze dans la péninsule Italienne. Mais les colons orientaux ont certainement transmis de nouvelles variétés et des améliorations culturales et ils ont dû développer ces cultures dans la partie nord de la Méditerranée.

\section{Séminaire 2 - La production d'huile d'olive dans la péninsule lbérique pendant la préhistoire et la protohistoire}

Isabel Bonora Andujar (ingénieur au musée du Louvre), le 14 novembre 2017

Par rapport aux autres produits de la fameuse triade méditerranéenne - céréales, vin et huile -, l'historiographie a établi une relation quasi axiomatique entre l'oléiculture et la péninsule Ibérique. Cette vision supportée aujourd'hui par la première place de l'Espagne dans la production mondiale d'huile se fonde sur les textes agronomiques latins montrant une grande production d'huile d'olive dès le $\mathrm{I}^{\text {er }}$ siècle après $\mathrm{J}$.-C. au moins. Une contradiction se fait jour entre les théories historiographiques «diffusionnistes » qui voient l'implantation de l'olivier et la diffusion de la culture oléicole dans la péninsule Ibérique à partir du $\mathrm{I}^{\mathrm{er}}$ millénaire avant J.-C., en les faisant coïncider avec l'arrivée des colons phéniciens, et les études archéobotaniques, qui défendent l'exploitation, voire la culture de l'olivier dans la péninsule au moins depuis le Néolithique. Ce séminaire a eu pour objectif de contester ces paradigmes en traitant des quatre questions suivantes: l'origine de l'olivier dans la péninsule Ibérique, les débuts de la production d'huile d'olive, l'action des Phéniciens et celle des Ibères.

\section{Séminaire 3 - Prospérité et décadence de l'oléiculture dans les Hautes Steppes tunisiennes dans l'Antiquité}

Samira Sehili (professeur à l'université de Tunis La Manouba), le 28 novembre 2017

Avec la domination romaine en Afrique, l'oléiculture connut un grand développement dû aux investissements réalisés par les Italiens dans les provinces conquises et par l'accroissement des exportations notamment vers l'Italie. La région du Haut-Tell dans le centre de la Tunisie se caractérise par de fortes densités de pressoirs à huile et par la concentration des installations les plus importantes par leur taille et leur technologie. On y trouve des huileries comptant jusqu'à douze pressoirs à Henchir El Begar au cours du IV siècle de notre ère. À partir du Ve siècle, l'huile d'olive perdit du terrain en Occident où elle fut relayée par les graisses animales. Sa production recula en Afrique du fait de la récession des provinces occidentales, du ralentissement considérable et des perturbations du commerce, de l'arrivée de nouvelles peuplades germaniques dont les habitudes de consommation étaient différentes de celles des Méditerranéens. Les grandes huileries dispersées dans les campagnes furent alors abandonnées et, probablement pour des raisons de sécurité, on rapatria les installations de pressurage à l'abri des remparts des villes telles 
Thuburbo Maius ou Uchi Maius. Les changements économiques de la fin de l'Antiquité ont eu raison de l'équilibre fragile qui avait permis l'essor sans précédent de l'oléiculture durant l'Empire romain.

\section{Séminaire 4 - L'oléiculture dans l'ouest de la province romaine d'Afrique}

Mariette de Vos (professeur à l'université de Trento, Italie), le 12 décembre 2017

En Tunisie la recherche archéologique a porté sur le bassin versant de la Medjerda, à $100 \mathrm{~km}$ de Tunis. La prospection a été systématique et à couverture totale, sur une bande de $64 \mathrm{~km}^{2}$ aux environs des cités Thugga et Téboursouk et à niveau extensif tout autour de la zone prospectée intensivement jusqu'à couvrir l'entière feuille 33 . On a fouillé stratigraphiquement l'établissement rural d'Aïn Ouassel, pour établir une chronologie de la poterie et pour retracer les conditions de vie et de travail des colons protagonistes de la lex hadriana dont une copie a été trouvée sur place.

En Algérie, le paysage et le travail ont été différents. La prospection extensive a été réalisée dans des régions le long de la côte et près de la frontière de Tunisie couvertes de chênes-lièges. Les sites antiques étaient donc plus difficiles à repérer et à dater mais ils étaient dans un état de conservation exceptionnel, notamment la villa romaine de Ksar Fatma préservée jusqu' au premier étage.

\section{RECHERCHE}

\section{FoUILLES ARCHÉOLOGIQUES DE LA COLONIE GRECQUE DE CUMES}

Cumes fait l'objet de fouilles plus ou moins continues et officielles depuis 1606. Mais à partir de 1994, sous l'impulsion de Stefano de Caro, alors surintendant de l'archéologie de Naples, un grand programme de recherche a été lancé pour mieux comprendre la topographie et la chronologie de ce site immense (110 ha). Parmi les acteurs de cette entreprise, aux côtés des universités de Naples, le Centre Jean Bérard a été chargé de reconnaître les abords de la ville, rechercher les ports, explorer les nécropoles et les sanctuaires extra-urbains. Il travaille depuis 2001 dans la zone située au nord des remparts, en bordure de la lagune, où se trouvent à la fois un sanctuaire grec, des routes et des sépultures s'étageant depuis le début du $\mathrm{I}^{\mathrm{er}}$ millénaire avant J.-C. jusqu'au $\mathrm{VI}^{\mathrm{e}}$ siècle après J.-C.

Les fouilles archéologiques françaises y sont dirigées par Jean-Pierre Brun (Collège de France) et Priscilla Munzi (Centre Jean Bérard, USR 3133 CNRS École française de Rome), avec le soutien financier du ministère de l'Europe et des Affaires étrangères (commission des fouilles), de l'École française de Rome et de la Fondation du Collège de France qui a donné les moyens d'élargir les dégagements et d'approfondir la recherche.

La cité antique de Cumes est située à $25 \mathrm{~km}$ à l'ouest de Naples, sur la côte de la mer Tyrrhénienne en face de l'île d'Ischia. Les historiens antiques considéraient Cumes comme l'une des toutes premières colonies grecques établies en Occident. Elle fut fondée dans la seconde moitié du VIII ${ }^{\mathrm{e}}$ siècle avant J.-C. par des Grecs venus d'Eubée. Elle connut rapidement une grande prospérité dont l'archéologie témoigne. Au fil des temps, elle eut de nombreuses vicissitudes surmontant une attaque des Étrusques en 524, puis en 474, succombant à l'avancée des Samnites en 423, entrant dans l'alliance de Rome, résistant à Hannibal en 217. Par la suite, après les guerres 
civiles de la fin de la République, elle fut très prospère durant l'Empire romain et subsista sans changements notables jusqu' aux guerres entre les Goths et les troupes de Justinien au milieu du $\mathrm{VI}^{\mathrm{e}}$ siècle de notre ère. Après un renouveau à l'époque byzantine, la cité entra en totale décadence et fut abandonnée au début du XIII siècle.

Les recherches de 2018 ont porté sur la nécropole gréco-romaine. Parmi des centaines de sépultures antiques, deux tombes à chambre ont été mises au jour. Elles sont constituées d'une construction voûtée enterrée en grand appareil de tuf volcanique local. La façade est percée d'une porte fermée par un gros bloc. À l'intérieur, se trouvent trois coffres funéraires qui contenaient les corps des défunts inhumés avec des offrandes qui permettent de les dater des $\mathrm{III}^{\mathrm{e}}$ et $\mathrm{II}^{\mathrm{e}}$ siècles avant J.-C. Certaines tombes étaient décorées de peintures dont celle découverte en juin 2018 qui montre un exceptionnel décor figuré, surtout conservé sur la paroi surmontant l'entrée. La partie visible représente, de gauche à droite, un trépied maintenant une amphore de vin à la verticale, une table et un grand vase. À droite de la porte, un jeune serviteur nu apporte une cruche de vin aux convives qui devaient être représentés sur les parois latérales. Un grand cratère à calice en bronze est peint à sa droite : c'est dans ce vase que le vin et l'eau étaient mélangés avant d'être servi aux convives. L'iconographie est relativement courante à partir de l'époque archaïque mais il est rare que des tombes de la période hellénistique conservent des décors de ce type.

\section{PuBLications}

Brun J.-P., Munzi P. et Pouzadoux C., «"Memorie dal sottosuolo" : una nuova tomba "a schiena" di età sannita nella necropoli di Cuma », in L. CICALA et B. FERRARA (dir.), "Kithon Lydios ». Studi di storia e archeologia con Giovanna Greco, Pouzzoles, Naus Editoria, 2017, p. 373-391.

BRUN J.-P., FAUCHER T. et REDON B., « An early Ptolemaic bath in the fortress of Bi'r Samut (Eastern desert) », in B. REDON (dir.), Collective Baths in Egypt. 2: New Discoveries and Perspectives, Le Caire, Presses de 1'IFAO, coll. «Études urbaines », nº 10, 2017, p. 13-23.

Cavassa L., Munzi P., Brun J.-P., Botte E., Germinario C., Grifa C., Mercurio M., LANGella A. et Morra V., «Cumes. Le matériel tardo-antique découvert dans un puits. Entre données typologiques et analyses archéométriques », in D. DIXNEUF (dir.), LRCW5 : Late Roman coarse wares, cooking wares and amphorae in the Mediterranean : Archaeology and archaeometry, Alexandrie, Centre d'études alexandrines, coll. «Études alexandrines », $\mathrm{n}^{\circ} 42$, vol. 1, 2017, p. 385-405.

Zanella S., Brun J.-P., Denoyelle M., Rouillard P. et Verger S. (dir.), Les Archives de fouilles : modes d'emploi, Paris, Collège de France, coll. «Institut des civilisations », 2017, DOI : 10.4000/books.cdf.4859.

Brun J.-P., Munzi P., Botte E., Morra V., Graziano Fabio S., Di Benedetto C., Guarino V., Cappelletti P. et Covolan M., «Cuma: Il monumento funerario della «Sfinge» (A63) nella necropoli della Porta mediana », in C. GASPARRI et C. CAPALDI (dir.), Complessi monumentali e arredo scultoreo nella Regio I Latium et Campania Nuove scoperte e proposte di lettura in contesto, Pouzzoles, Naus Editoria, 2017, p. 9-31.

Brun J.-P., Munzi P., Cavassa L., Chapelin G., Covolan M., Duneufuardin P., Le Berre S., Lemaire B., Leone M., Mailleur S., MeluziIs N., Neyme D. et Watel A., «Recherches archéologiques à Cumes (Campanie, Naples)», Chronique des activités archéologiques de l'École française de Rome, 2015, DOI : 10.4000/cefr.1444.

Brun J.-P., Munzi P., Chapelin G., Covolan M., Lemaire B., LeOne M., Meluzis N. et SACHAU-CARCEL G., «Recherches archéologiques dans la nécropole de la Porte médiane à 
Cumes : Campagne $2016 »$, Chronique des activités archéologiques de l'École française de Rome, 2017, DOI : 10.4000/cefr.1786.

Barillot M., Berre M., Boisson A., Botte E., Brun J.-P., CongÈs G., LeguillouX M. et MiChel J.-M., «L'atelier de potiers du Collet Long: La Crau, Var», Revue du Centre Archéologique du Var, vol. 2015/2016, 2017, p. 107-125.

BRUN J.-P., « Archéologie des techniques de l'Antiquité », in J.-P. DEMOULE, D. GARCIA et A. SCHNAPP (dir.), Une histoire des civilisations: comment l'archéologie bouleverse nos connaissances, Paris, La Découverte/Inrap, 2018, p. 559-563.

BRUn J.-P., «Chronologie des forts des routes de Myos Hormos et de Bérénice durant la période gréco-romaine », in T. FAUCHER, B. REdon et S. Sidebotham (dir.), Le Désert oriental d'Égypte durant la période gréco-romaine : bilans archéologiques, Paris, Collège de France, coll. «Institut des civilisations », 2018, http://books.openedition.org/cdf/5155.

BRUN J.-P., FAUCher T., REdON B. et Sidebotham S., «Introduction », in T. FAUCher, B. REDOn et S. Sidebotham (dir.), Le Désert oriental d'Égypte durant la période grécoromaine : bilans archéologiques, Paris, Collège de France, coll. «Institut des civilisations », 2018, http://books.openedition.org/cdf/5136.

Brun J.-P., Faucher T., Redon B. et Sidebotham S. (dir.), Le Désert oriental d'Égypte durant la période gréco-romaine: bilans archéologiques, Paris, Collège de France, coll. «Institut des civilisations », 2018, DOI : 10.4000/books.cdf.4932 [hal-01757996]. 